\title{
Role of combination bortezomib and pegylated liposomal doxorubicin in the management of relapsed and/or refractory multiple myeloma
}

\author{
Jatin J Shah' \\ Robert Z Orlowski ${ }^{1,2}$ \\ Sheeba K Thomas' \\ 'Departments of Lymphoma/Myeloma; \\ ${ }^{2}$ Experimental Therapeutics, Division \\ of Cancer Medicine, University of \\ Texas MD Anderson Cancer Center, \\ Houston, Texas, USA
}

\begin{abstract}
The first in class proteasome inhibitor bortezomib (B) received its initial regulatory approval for therapy of patients with multiple myeloma (MM) in the relapsed/refractory setting. Modulation of proteasome function, however, is also a rational strategy for chemosensitization, and a variety of agents have shown synergistic activity with bortezomib pre-clinically, including anthracyclines. This formed the basis for evaluation of a regimen of bortezomib with pegylated liposomal doxorubicin (PLD). PLD+B, in a phase I study, induced a predictable and manageable toxicity profile, and showed encouraging anti-MM activity. In a recent international, randomized phase III trial, PLD+B demonstrated a superior overall response rate and response quality compared to bortezomib alone, as well as a longer time to progression, duration of response, progression-free survival, and overall survival. Sub-analyses revealed benefits in almost all clinically relevant subgroups, including several which would be considered to have high-risk disease. These findings have led to the establishment of the PLD+B regimen as one of the standards of care for patients with relapsed and/or refractory myeloma. Efforts are now underway to build on this combination further by adding other active anti-myeloma agents. In this review, we will discuss the role of PLD+B as an important addition to our therapeutic armamentarium for patients with MM.
\end{abstract}

Keywords: multiple myeloma, relapsed/refractory, pegylated liposomal doxorubicin, bortezomib, Doxil $^{\circledR}$, Velcade ${ }^{\circledR}$

\section{Introduction to management strategies incorporating bortezomib and anthracyclines in multiple myeloma}

In May 2003, bortezomib (B) (Velcade ${ }^{\circledR}$; Millenium Pharmaceuticals), a first in class proteasome inhibitor, received accelerated approval by the Food and Drug Administration (FDA) for use in patients with multiple myeloma (MM) who had received at least 2 prior lines of therapy, and had progressive disease on the most recent therapy. ${ }^{1}$ In March 2005, this approval was amended to allow the use of bortezomib for patients with MM after 1 or more prior lines of therapy. ${ }^{2}$ In the relapsed or primary refractory setting, single-agent bortezomib has provided response rates of $27 \%$ to $43 \%$, with median times to progression of 6.2 to 7.0 months. ${ }^{3-5}$ To improve these outcomes, combinations of bortezomib with other agents active in MM have been explored. Among these drug classes are anthracyclines, whose efficacy in treating myeloma was established with the combination of vincristine-doxorubicin-dexamethasone in the 1980s. ${ }^{6-11}$ Pre-clinical studies combining bortezomib and doxorubicin have demonstrated the ability of each drug to reduce chemoresistance to the other, and indeed these drugs have shown synergy in combination. 
Unfortunately, the administration of continuous infusion doxorubicin is complicated by the requirement for central line placement which increases the risks of infections and thrombosis, while doxorubicin itself is associated with the risks of neutropenia and cardiotoxicity. In an effort to maintain the efficacy of doxorubicin, while improving its tolerability, a pegylated, liposomal form of doxorubicin (PLD, Doxil ${ }^{\circledR}$; Johnson and Johnson) was developed. With a half-life of approximately 79 hours, a single 60- to 90-minute administration of pegylated liposomal doxorubicin is comparable in efficacy to continuous infusion doxorubicin, but has a lower incidence of neutropenia, alopecia and cardiac toxicity. ${ }^{12,13}$

The pre-clinical synergy observed between proteasome inhibitors and anthracyclines led to the development of clinical trials investigating the use of these drug classes in combination. In this review, we discuss treatment strategies using bortezomib together with PLD in patients with relapsed and refractory MM.

\section{Mechanism of action Doxorubicin}

Doxorubicin, an anti-tumor anthracycline antibiotic produced by the soil fungus Streptomyces peucetius, has been used in a number of malignancies including MM. Its cytotoxicity is thought to result in large part from inhibition of the DNA repair enzyme, topoisomerase II. ${ }^{14,15}$ It also intercalates into double-stranded DNA, producing structural changes that interfere with DNA and RNA synthesis. ${ }^{16-18}$ Doxorubicin and other anthracyclines also chelate iron, thereby generating reactive oxygen species, such as oxygen free radicals, hydroxyl radicals, and hydrogen peroxide, that cleave DNA, messenger mRNA, proteins, and lipids. The formation of oxygen free radicals and the peroxidation of lipids also contribute to the cardiac toxicity that is characteristic of non-liposomal anthracyclines..$^{19,20}$

\section{Bortezomib}

Bortezomib is a dipeptide boronic acid analog which targets the ubiquitin-proteasome pathway (UPP) by inhibiting the function of the $26 \mathrm{~S}$ proteasome complex. Inhibition of the UPP blocks protein and transcription factor degradation, leading to accumulation of misfolded/damaged proteins, and inducing heat shock stress responses within the cell..$^{21,22}$ Bortezomib mediates its cytotoxic effects via alterations in multiple pathways including inhibition of nuclear factor

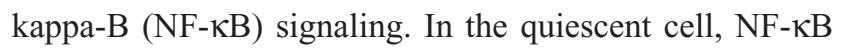
is bound to the inhibitory protein $\mathrm{I} \kappa \mathrm{B}$, rendering it inactive. However, with phosphorylation, ubiquitination, and degradation of IKB by the ubiquitin-proteasome pathway, $\mathrm{NF}-\mathrm{\kappa B}$ is released from the bound state and translocates from the cytoplasm to the nucleus. Activated NF- $\mathrm{KB}$ initiates an immune/inflammatory response via increased gene transcription of multiple cytokines, and also contributes to cellular resistance to apoptosis and chemotherapy resistance. Bortezomib inhibits I $\kappa B$ degradation, thereby suppressing NF- $\kappa B$ activity and contributing to apoptosis. ${ }^{21,23-26}$ Bortezomib's anti-tumor activity is also mediated by a number of proapoptotic pathways including accumulation of p53, p21 and p27, activation of c-Jun N-terminal kinase (JNK), inhibition of the interleukin (IL)-6-induced Ras/Raf/mitogen-activated protein kinase (MAPK) pathway, and interference with the anti-apoptotic effects of Bcl-2. ${ }^{26-32}$ Additionally, preclinical studies with bortezomib have demonstrated a reduction in levels of cytokines (IL-6), angiogenesis factors (vascular endothelial growth factor), and growth factors (insulin-like growth factor-1), which among other effects inhibits adhesion of myeloma cells to bone marrow stromal cells..$^{23,25,26,28}$

\section{Rationale for combination}

One of the mechanisms of doxorubicin resistance is via up-regulation of NF- $\kappa \mathrm{B}$ expression. ${ }^{31,32}$ As bortezomib suppresses the activity of NF- $\mathrm{KB}$, chemo-resistance to PLD induced by this mechanism would be abrogated by using these drugs in combination. ${ }^{29}$ Bortezomib may also sensitize myeloma cells to DNA damage by down-regulating the transcription of DNA repair enzymes, as demonstrated in in vitro studies as well as by gene expression profiling. ${ }^{30}$ P-glycoprotein, encoded in the multi-drug resistance gene 1 (MDR1), functions as a cell membrane transporter responsible for the efflux of toxins including multiple chemotherapy agents. ${ }^{6,27}$ Preliminary data suggest proteasome inhibition decreases expression of P-glycoprotein, and subsequently bypasses a well-defined mechanism of multi-drug resistance. ${ }^{33}$ Cell adhesion mediated drug resistance (CAM-DR) also contributes to the development of resistance to conventional chemotherapeutics, and in addition to suppression of NF- $\kappa$ B activity, bortezomib may overcome CAM-DR. ${ }^{30}$

Anthracyclines, in turn, may also overcome bortezomib resistance by inhibiting the mitogen-activated protein kinase phosphatase (MPK-1) pathway. One of several mechanisms of proteasome inhibitor-induced apoptosis is via activation of JNK. However, bortezomib induces MPK-1, which can dephosphorylate and inactivate JNK. Anthracyclines have been shown to inhibit MPK-1 activity, potentially contributing to the synergy of doxorubicin and bortezomib. ${ }^{34}$ Combination studies of bortezomib and anthracyclines in in vitro 
and in vivo models have demonstrated improved inhibition of MPK-1, increased activation of JNK, and increased apoptosis. These preclinical observations led investigators to develop clinical trials evaluating the combination of bortezomib and PLD. ${ }^{30}$

\section{Pharmacology/pharmacokinetics}

In a phase I study of PLD+bortezomib (PLD+B), the pharmacodynamics of bortezomib were evaluated using an ex vivo assay of 20S-proteasome inhibition by measuring the chymotryptic proteasome protease activity. ${ }^{35}$ Proteasome inhibition on Days 1 and 4 prior to PLD administration was $63.7 \%$, and with the addition of PLD on days 8 and 11 , mean proteasome inhibition increased to $67.4 \%$. As might be expected, proteasome inhibition was lowest at the starting bortezomib dose of $0.9 \mathrm{mg} / \mathrm{m}^{2}$, with no difference noted between the 1.05 and $1.50 \mathrm{mg} / \mathrm{m}^{2}$ dose levels.

The $t_{1 / 2}$ of PLD was 80 hours and remained constant across escalating bortezomib dose cohorts; suggesting no significant alteration in the pharmacokinetic and pharmacodynamic profile of bortezomib and PLD when combining the two agents. ${ }^{35}$

\section{Efficacy studies}

The combination of bortezomib and pegylated liposomal doxorubicin was first evaluated clinically in a phase 1 study led by Orlowski et al with 42 patients having any confirmed hematologic malignancy. Notably, the majority $(n=24)$ had $\mathrm{MM} .{ }^{35}$ Dose reductions and treatment delays were frequent at the 1.5 and $1.6 \mathrm{mg} / \mathrm{m}^{2}$ dose levels of bortezomib, and therefore $1.3 \mathrm{mg} / \mathrm{m}^{2}$ of bortezomib on days $1,4,8$, and 11 in combination with $30 \mathrm{mg} / \mathrm{m}^{2}$ of PLD on day 4 of a 21-day cycle was established as the optimal dosing regimen. Among 22 evaluable patients with MM, 36\% achieved either complete remission (CR) or near CR, and another 36\% achieved partial remission (PR). Prior to study enrollment, 13 patients had received anthracyclines to which their disease had either not responded, or initially responded but then derived no further benefit; among them, 38\% achieved CR or near CR, and another $23 \%$ achieved PR. With extended follow-up, the median time to disease progression (TTP) among the 22 evaluable patients was 9.3 months, with a median time to re-treatment (TTRT) of 24.2 months, and median overall survival of 38.3 months. Both the median TTP and median TTRT compared favorably to outcomes seen on the patients' prior therapy. ${ }^{35,36}$ This experience led to a prospective, international, randomized, controlled phase III trial led by Orlowski et al comparing single-agent bortezomib with the combination of PLD+B in patients with relapsed or primary refractory MM. ${ }^{37}$ The results of this trial led the FDA to approve combination therapy with PLD+B in May 2007, for the treatment of bortezomib-naïve patients who had progressed after at least 1 prior line of therapy. ${ }^{38}$ In this study, 646 patients, who had progressed after $\geq 1$ line of therapy and were bortezomib-naïve, were enrolled and stratified according to $\beta_{2}$-microglobulin and response to prior therapy (relapsed vs refractory). Patients on both arms were treated with $1.3 \mathrm{mg} / \mathrm{m}^{2}$ of bortezomib on days $1,4,8$ and 11 on a 21 day cycle, while patients on the combination arm also received $30 \mathrm{mg} / \mathrm{m}^{2}$ of PLD on day 4 of each cycle. Patients were treated for up to 8 cycles or until complete response, disease progression, or unacceptable toxicity developed, though patients tolerating therapy were allowed to continue beyond 8 cycles if they showed continued disease response. The primary endpoint was TTP on an intent-to-treat population, while secondary endpoints were overall survival (OS), overall response rate (complete response + partial response) as defined by the European Blood and Marrow Transplant Group criteria, and safety profile. ${ }^{39}$

Among patients treated with the combination of PLD+B, TTP was higher at 9.3 months, compared with 6.5 months among patients treated with bortezomib alone $(\mathrm{p}=0.000004)$. Progression-free survival (PFS) was also longer in those treated on the combination arm than in those treated with bortezomib monotherapy ( 9.0 vs 6.5 months, $p=0.000026$ ). An increase in the duration of response (DOR) from 7.0 to 10.2 months with combination therapy paralleled the improvements seen in TTP and PFS. Early follow-up of survival data also suggested an improvement in 15-month OS among those treated with PLD+B compared with those treated with bortezomib alone $(76 \%$ vs $65 \%, \mathrm{p}=0.03)$. By intent-to-treat analysis, the overall response rate (ORR) was similar between monotherapy (41\%) and combination therapy arms $(44 \%)(p=0.43)$; however, rates of CR + very good partial response (VGPR) were higher (19\% vs 27\%) with combination therapy $(\mathrm{p}=0.0157)$. When only responseevaluable patients were included in the analysis, the overall response rate for bortezomib alone $(n=310)$ was $44 \%$ and that for PLD+B $(n=303)$ was $52 \%(p=0.05) .{ }^{37}$

Braccalenti et al have taken an alternative approach to evaluating the effect of adding PLD to the anti-tumor activity of B. In an ongoing study, $40 \mathrm{mg} / \mathrm{m}^{2}$ of PLD given once every 21 days is added to bortezomib $1.3 \mathrm{mg} / \mathrm{m}^{2}$ (on days $1,4,8,11$ for the first 8 cycles, and then on days 1, 8, 15, and 22) after either 8 cycles (cohort 1) or 3 cycles (cohort 2 ) of therapy. Among 5 patients enrolled in cohort 1, 4 had 
objective responses with bortezomib and 1 had disease stabilization; the addition of PLD did not improve the quality of the responses achieved with bortezomib alone. In cohort 2, 9 of 17 evaluable patients had objective responses (52.9\%) after 3 cycles of bortezomib monotherapy. Of these, 7 patients had conversion of their remission from either PR (4 patients) or near CR (3 patients) to CR. Though we await further follow-up of this study, its preliminary results provide further evidence that adding PLD to bortezomib enhances activity of the latter in certain patients. ${ }^{40}$

\section{Sub-group analyses from randomized phase III trial}

A number of formal subgroup analyses were performed to compare the efficacy of PLD+B with bortezomib alone among elderly patients, those with high-risk disease, those having prior autologous stem cell transplant (ASCT) and early relapse after ASCT, those with previous anthracycline and thalidomide exposure, and those having one or multiple prior lines of therapy (Table 1). As described below, these subgroups all benefited from combination therapy. Subgroups that did not benefit from combination therapy were those with low $\beta_{2}$-microglobulin and those with chromosome 13 deletion.

In a pre-specified subgroup analysis of 250 patients $\geq 65$ years of age, the addition of PLD improved TTP from 205 days to 276 days ( $\mathrm{p}=0.0056, \mathrm{HR}=1.82,1.18-2.79$ ) and also improved DOR from 227 days to 311 days. ORR was also increased from $39 \%$ to $49 \%$ by combination therapy. The benefit of adding PLD in this older patient population was comparable to that seen in younger patients. ${ }^{41}$

A second pre-specified subgroup analysis evaluated the effect of adding PLD to bortezomib in patients with high-risk disease, where high-risk disease was defined as having either an elevated $\beta_{2}$-microglobulin $(\geq 5.5 \mathrm{mg} / \mathrm{L})$ or primary refractory disease. TTP was significantly increased in the combination therapy arm, both among patients having an elevated $\beta_{2}$-microglobulin (178 vs 276 days; $\mathrm{p}=0.0007)$, as well as among those having primary refractory disease (189 days vs median not reached, $\mathrm{p}=0.0322$ ). Patients with an elevated $\beta_{2}$-microglobulin also showed improvement in ORR (33\% vs 49\%), and DOR (211 vs 310 days). ${ }^{42}$

The third pre-specified subgroup analysis compared patients with and without a history of prior ASCT. In patients without a prior history of ASCT, TTP increased from 197 to 331 days ( $\mathrm{p}=0.0009$ ) with the addition of PLD, ORR improved from $39 \%$ to $45 \%$, and DOR increased from 204 days to 394 days. Similar benefits in TTP (197 vs 276 days, $\mathrm{p}=0.0011$ ) and DOR (227 days vs 309 days) were also observed in patients who had had a prior $\mathrm{ASCT}{ }^{43}$

Table I Subgroup analyses from phase III trial of bortezomib vs pegylated liposomal doxorubicin + bortezomib (PLD+B)

\begin{tabular}{|c|c|c|c|c|c|}
\hline \multirow[t]{2}{*}{ Subgroup } & \multicolumn{2}{|c|}{ ORR (\%) } & \multicolumn{2}{|c|}{ TTP (days) } & \multirow[t]{2}{*}{ p value } \\
\hline & B & PLD+B & B & PLD+B & \\
\hline Age $\geq 65$ yrs & 39 & 49 & 205 & 276 & 0.0056 \\
\hline$B_{2}$-microglobulin $\geq 5.5 \mathrm{mg} / \mathrm{L}$ & 33 & 49 & 178 & 276 & 0.0007 \\
\hline Primary refractory disease & 48 & 43 & 189 & MNR & 0.0322 \\
\hline No prior ASCT & 39 & 45 & 197 & 331 & 0.0009 \\
\hline Prior ASCT & 46 & 50 & 197 & 276 & 0.0011 \\
\hline$\geq 2$ prior lines of therapy & & $\mathrm{N} / \mathrm{A}$ & 186 & 276 & $<0.0001$ \\
\hline I prior line of therapy & & $\mathrm{N} / \mathrm{A}$ & 199 & 330 & 0.036 \\
\hline Anthracycline naïve & & $\mathrm{N} / \mathrm{A}$ & 197 & 282 & 0.015 \\
\hline Anthracycline exposed & & N/A & 197 & 295 & 0.0001 \\
\hline ISS stage 3 & & $\mathrm{~N} / \mathrm{A}$ & 177 & 276 & $<0.0007$ \\
\hline ISS stage 2 & & $\mathrm{~N} / \mathrm{A}$ & 186 & 250 & $<0.004$ \\
\hline ISS stage I & & $\mathrm{N} / \mathrm{A}$ & 223 & 331 & 0.067 \\
\hline TSD $>2$ years & & $\mathrm{N} / \mathrm{A}$ & 197 & 295 & $<0.0001$ \\
\hline $\mathrm{TSD} \leq 2$ years & & $\mathrm{N} / \mathrm{A}$ & 197 & 243 & 0.0033 \\
\hline Relapse $<12$ months post & 74 & 93 & 205 & 276 & 0.13 \\
\hline ASCT & & $(p=0.01)$ & & & \\
\hline
\end{tabular}

Abbreviations: ASCT, autologous stem cell transplant; ISS, International Staging System; MNR, median not reached; N/A, not available; TSD, time since diagnosis; TPP, time to disease progression; ORR, overall response rate. 
Bladé et al also presented a pre-specified analysis to assess the efficacy of combination therapy with PLD+B based on the number of prior lines of therapy and previous anthracycline exposure. The TTP was similar with PLD+B between those who had $\geq 2$ lines of prior therapy ( 276 days) vs 1 prior therapy ( 330 days) (heterogeneity test, $p=0.523$ ), as well as between those who were anthracycline-exposed (median $144 \mathrm{mg} / \mathrm{m}^{2}$ in both treatment arms) or -naïve (heterogeneity test, $\mathrm{p}=0.716$ ). The incidence of symptomatic cardiac events was also comparable between treatment arms in each subgroup. There was a uniform benefit with PLD+B combination therapy in TTP regardless of the number of prior lines of therapy and anthracycline exposure. ${ }^{44}$

The impact of treatment with prior thalidomide on outcomes of patients treated with PLD+B was also evaluated in a post hoc analysis of the phase III study by Sonneveld et al. Prior exposure to thalidomide did not affect TTP (295 days for thalidomide-naïve vs 270 days for thalidomide-exposed) or DOR (310 days for thalidomide-naïve vs 319 days for thalidomide-exposed). ORR was also comparable between the thalidomide-naïve and -exposed arms (48\% vs $47 \%){ }^{45}$ A second post hoc analysis by Sutherland et al evaluated the effect of International Staging System (ISS) disease stage and time since initial diagnosis (TSD; 2 cohorts: $>2$ years and $\leq 2$ years) on outcomes of patients treated with PLD+B. There was a consistent benefit in TTP across ISS stages $2(\mathrm{HR}=1.8$, $\mathrm{p}<0.004)$ and $3(\mathrm{HR}=2.1, \mathrm{p}<0.007)$ but not ISS stage 1 $(\mathrm{HR}=1.6, \mathrm{p}=0.067)$. TSD did not affect the benefit in TTP $(>2$ years, HR 1.83; $\leq 2$ years, $\mathrm{HR}=1.75) .{ }^{46}$

A post hoc analysis of outcomes of patients, who had an early relapse ( $<12$ months) after ASCT, was also performed (Kumar et al). Among these patients, the 12-month post-randomization survival was significantly higher in those treated with combination therapy (93\% vs $74 \%, \mathrm{p}=0.01)$. TTP in the early relapse cohort also suggested improvement with combination therapy (205 vs 276 days), but did not reach statistical significance ( $p=0.13$, HR $=0.94$ ). A similar benefit in TTP was noted among patients whose myeloma relapsed 12 months or later after ASCT, suggesting that the benefits of treatment with combination therapy were not affected by timing of relapse after ASCT. No differences in ORR (CR+PR) or VGPR rates were seen in patients with early vs late relapses, or between treatment arms within each group. ${ }^{47}$

\section{Safety/tolerability}

Based on the risk of dose reductions and delays at higher dose levels of bortezomib (PLD was maintained at a fixed dose level) during the phase I study of PLD+B, a dosing schedule of bortezomib $1.3 \mathrm{mg} / \mathrm{m}^{2}$ on days $1,4,8$, and 11 , and PLD $30 \mathrm{mg} / \mathrm{m}^{2}$ on day 4 on a 21-day cycle was determined to be the most feasible for multiple cycle administration. ${ }^{35}$ Neutropenia and thrombocytopenia were the most common reasons for therapy-related dose delays, followed by diarrhea and fatigue. ${ }^{35}$

In the phase I study, $43 \%$ of patients experienced $\geq$ grade 3 thrombocytopenia, and $40 \%$ and $17 \%$ respectively experienced $\geq$ grade 3 lymphopenia and neutropenia. The most notable infectious complications were febrile neutropenia (10\%) and pneumonia (14\%). ${ }^{35}$ By comparison, $23 \%$ of patients in the PLD+B arm of the phase III study developed $\geq$ grade 3 thrombocytopenia and $29 \%$ had $\geq$ grade 3 neutropenia (Table 2). Though the incidence of $\geq$ grade 3 neutropenia was nearly twice that seen among those treated with bortezomib alone (15\%), the incidence of febrile neutropenia was comparable between study arms ( $2 \%$ vs $3 \%$ ). Rates of thrombocytopenia were also similar. ${ }^{37}$

Peripheral neuropathy (PN) is one of the most common toxicities of single-agent bortezomib, with an incidence of grade $1-4$ events seen in $36 \%$ of patients and grade $3-4$ events in $8 \%$ in the APEX trial. ${ }^{4}$ In the phase I study of PLD+B, where doses of bortezomib up to $1.5 \mathrm{mg} / \mathrm{m}^{2}$ were evaluated, an incidence of 55\% (12\% grade 3-4) was observed..$^{35}$ However, in the phase III study of bortezomib vs PLD+B, where $1.3 \mathrm{mg} / \mathrm{m}^{2}$ of bortezomib was given to all patients, rates of peripheral neuropathy were comparable to those seen in previous clinical trials of single-agent bortezomib. ${ }^{37}$ Rates of peripheral neuropathy were also comparable between treatment arms of this study (PN grades 1-4: 39\% and 35\% respectively; $\geq$ grade $3: 9 \%$ and $4 \%$ ), despite an equivalent number of median cycles of therapy $(\mathrm{n}=5)$ and similar cumulative dose of bortezomib (monotherapy, $24.4 \mathrm{mg} / \mathrm{m}^{2}$; combination, $23.2 \mathrm{mg} / \mathrm{m}^{2}$ ). This suggests that PLD added no further neurotoxicity to bortezomib. ${ }^{37}$

Reducing the potential for cardiac toxicities with doxorubicin was one of the most important reasons for the development of PLD. Data from the phase III study of bortezomib vs PLD+B showed that $7 \%$ and $9 \%$ of patients respectively had a fall in their left ventricular ejection fraction by $\geq 15 \%$, suggesting that PLD does not add significant risk of cardiotoxicity to bortezomib. Congestive heart failure was seen in $3 \%$ of patients on each treatment arm. ${ }^{37}$ In addition, in a pre-specified analysis by Bladé et al evaluating the role of prior anthracycline exposure, there was no increase in the incidence of symptomatic cardiac events with prior anthracycline exposure. ${ }^{44}$ 
Table 2 Grade 3-4 adverse events in phase III trial of bortezomib vs pegylated liposomal doxorubicin + bortezomib (PLD+B)

\begin{tabular}{|c|c|c|c|}
\hline Adverse event & Bortezomib (\%) $\mathbf{N}=318$ & PLD+B (\%) N = 318 & p value \\
\hline All drug-related adverse events & $165(52)$ & $217(68)$ & $<0.001$ \\
\hline Thrombocytopenia & $49(16)$ & 7I (23) & 0.249 \\
\hline Neutropenia & $46(15)$ & $94(29)$ & $<0.001$ \\
\hline Febrile neutropenia & $2(2)$ & $3(3)$ & $\mathrm{n} / \mathrm{a}$ \\
\hline Peripheral neuropathy & $39(9)$ & $35(4)$ & $\mathrm{n} / \mathrm{a}$ \\
\hline Cardiotoxicity & $7(3)$ & $10(2)$ & $\mathrm{n} / \mathrm{a}$ \\
\hline Thromboembolic events & $\mathrm{I}(\mathrm{I})$ & $I(I)$ & $\mathrm{n} / \mathrm{a}$ \\
\hline Diarrhea & $14(4)$ & $22(7)$ & 0.034 \\
\hline Palmar plantar erythrodysesthesia & $0(0)$ & $15(5)$ & $<0.001$ \\
\hline
\end{tabular}

In the phase III study, the addition of PLD to bortezomib resulted in a statistically significant increase in the incidence of nausea, vomiting, and diarrhea. ${ }^{37}$ Diarrhea (10\%), bowel obstruction (7\%), and lower gastrointestinal bleeding (5\%) were also seen in the phase I study of PLD+B. ${ }^{35}$ Finally, palmar plantar erythrodysesthesia, now known as hand-foot syndrome, was noted in $21 \%$ of patients (all grade $1-2$ ) in the phase I study and $16 \%$ of patients in the phase III study. ${ }^{35,37}$ It required discontinuation of PLD in 5\% of patients on the latter study.

In subset analyses of the phase III study, the effect of age, prior stem cell transplant, and high-risk disease on the incidence of adverse events was evaluated. ${ }^{41-43}$ With the exception of an increased incidence of grade $\geq 3$ neutropenia among patients $\geq 65$ years old, age did not impact the development of adverse events with combination therapy. Similarly, having primary refractory myeloma, a history of prior ASCT, or a serum $\beta_{2}$-microglobulin $\geq 5.5 \mathrm{mg} / \mathrm{L}$, did not affect rates or severity of adverse events encountered with PLD+B. ${ }^{42,43}$

\section{PLD+B-based combinations in relapsed/refractory disease (Table 3)}

Given the established activity of dexamethasone in combination with bortezomib as well as anthracyclines, Jakubowiak et al evaluated the addition of $40 \mathrm{mg}$ of dexamethasone on days $1-4$ to $\mathrm{PLD}+\mathrm{B}$ in the relapsed/refractory setting. ${ }^{48}$ In this study, patients were treated on a 21-day cycle of therapy for up to 6 cycles. Among 18 evaluable patients, who had received a mean of 3.6 prior regimens, the ORR was $55 \%$, and $33 \%$ achieved CR/nCR. Grade 3/4 neutropenia and thrombocytopenia were each seen in 7 patients; pneumonitis was seen in 5 patients and, unfortunately, resulted in 1 death. ${ }^{48}$

The combination of thalidomide-PLD-dexamethasone (ThaDD) has also provided noteworthy results, with an
ORR of $75.5 \%$, and CR rate of $30 \%$ in the relapsed/refractory setting. ${ }^{49}$ Building on this regimen, Offidani et al sought to improve these response rates further by adding bortezomib (ThaDD-V). In the induction phase of this feasibility study, patients received $100 \mathrm{mg}$ of thalidomide daily with PLD $30 \mathrm{mg} / \mathrm{m}^{2}$ iv on day 4 , dexamethasone $20 \mathrm{mg}$ on days 1 to 2,4 to 5,8 to 9,15 to 16 , and bortezomib $1.3 \mathrm{mg} / \mathrm{m}^{2}$ on days $1,4,8$, and 11 of every 28 days. Patients then alternated taking bortezomib $1 \mathrm{mg} / \mathrm{m}^{2}$ iv days $1,8,15$ with dexamethasone $20 \mathrm{mg}$ on days 1 to 2 , 8 to 9,15 to 16 , and thalidomide $100 \mathrm{mg}$ daily with dexamethasone $40 \mathrm{mg}$ on days 1 to 4 , every other cycle for a total 6 cycles, as consolidation therapy. Finally, patients received thalidomide $100 \mathrm{mg}$ daily as a maintenance regimen until disease relapse. The ORR seen in 20 evaluable patients was $80 \%$, of which $40 \%$ achieved either CR or stringent CR.${ }^{50}$ However, as 9 of the 20 patients developed grade $2-3$ peripheral neuropathy, the authors subsequently amended their protocol to reduce the dose intensity of both bortezomib and thalidomide. ${ }^{50,51}$

Chanan-Khan et al have also evaluated the combination of thalidomide, B, and PLD, but with a steroid-sparing approach. In this phase II study, patients were given $1.3 \mathrm{mg} / \mathrm{m}^{2}$ of bortezomib on days $1,4,15$, and 18 together with $20 \mathrm{mg} / \mathrm{m}^{2}$ PLD on days 1 and 15; $200 \mathrm{mg}$ thalidomide was given daily with low-dose warfarin prophylaxis. Among 17 evaluable patients, the ORR was $65 \%$, and CR rate was $23 \%$; PFS was 10.9 months. Among this heavily pre-treated population, responses were seen regardless of the prior therapies that patients had received. Grade 2 palmar plantar erythrodysesthesia developed in 2 patients, and 1 patient had grade 3 cellulitis. Importantly, no thromboembolic events were noted with this combination; and no patients developed evidence of cardiotoxicity, regardless of exposure to prior anthracyclines. ${ }^{52}$ 
Table 3 Pegylated liposomal doxorubicin + bortezomib combinations in relapsed/refractory setting

\begin{tabular}{|c|c|c|c|c|c|c|c|}
\hline Author & $\begin{array}{l}\text { Phase } \\
\text { of study }\end{array}$ & Regimen & Dose & $\begin{array}{l}\text { Evaluable } \\
\text { pts (\#) }\end{array}$ & PR (\%) & CR/nCR (\%) & $\begin{array}{l}\text { PFS/TTP } \\
\text { (med. months) }\end{array}$ \\
\hline Orlowski ${ }^{22}$ & 1 & B-PLD & $\begin{array}{l}\text { B } 0.9-1.5 \mathrm{mg} / \mathrm{m}^{2} \mathrm{~d} \mathrm{I}, 4,8, \mathrm{II} \\
\text { PLD } 30 \mathrm{mg} / \mathrm{m}^{2} \mathrm{~d} 4\end{array}$ & 22 & 36 & 36 & 9.3 (TTP) \\
\hline Orlowski ${ }^{22}$ & III & $B$ vs B-PLD & $\begin{array}{l}\text { B I } .3 \mathrm{mg} / \mathrm{m}^{2} \text { d I, } 4,8, \mathrm{II} \pm \text { PLD } \\
30 \mathrm{mg} / \mathrm{m}^{2} \text { d } 4\end{array}$ & 646 & $\begin{array}{l}4 \text { I vs } 44 \\
\text { (ORR) }\end{array}$ & $10 \mathrm{vs} 13$ & $\begin{array}{l}6.5 \text { vs } 9.3 \text { (TTP) } \\
6.5 \text { vs } 9.0 \text { (PFS) }\end{array}$ \\
\hline Chanan-Khan ${ }^{52}$ & ॥ & B-PLD + T & $\begin{array}{l}\text { B I. } 3 \mathrm{mg} / \mathrm{m}^{2} \mathrm{~d} \text { I, 4, I5, I8 PLD } \\
20 \mathrm{mg} / \mathrm{m}^{2} \text { d I, I5 T } 200 \mathrm{mg} \text { daily }\end{array}$ & 17 & 42 & 23 (all CR) & 10.9 (PFS) \\
\hline Jakubowiak ${ }^{48}$ & ॥ & B-PLD + D & $\begin{array}{l}\text { B I. } 3 \mathrm{mg} / \mathrm{m}^{2} \text { d I, } 4,8, \text { I I PLD } \\
30 \mathrm{mg} / \mathrm{m}^{2} \text { d } 4 \text { D } 40 \mathrm{mg} \text { d I-4 }\end{array}$ & 18 & 22 & 33 & $\mathrm{~N} / \mathrm{A}$ \\
\hline Chari $^{53}$ & $\mathrm{I} / \mathrm{II}$ & $B-P L D+M$ & $\begin{array}{l}\text { B } 0.7-I .0 \mathrm{mg} / \mathrm{m}^{2} \mathrm{~d} \mathrm{I}, 4 \text {, } \\
8, \text { I I PLD I0-20 mg/m² } \\
\text { d I M } 5-10 \mathrm{mg} / \mathrm{m}^{2} \mathrm{~d} \mathrm{I}\end{array}$ & 4 & 0 & 25 & $N / A$ \\
\hline
\end{tabular}

Abbreviations: $B$, bortezomib; D, dexamethasone; M, melphalan; PLD, pegylated liposomal doxorubicin; $T$, thalidomide; PR, partial response; CR, complete response; $\mathrm{nCR}$, near complete response; ORR, overall response rate; PFS, progression-free survival; TTP, time to disease progression.

Presently, Chari et al are evaluating the addition of melphalan to $\mathrm{PLD}+\mathrm{B}$ in a phase I/II dose escalation trial. ${ }^{53}$ Based on preliminary data from this study, 4 evaluable patients have been enrolled and treated at the first dose level, on which they have received PLD $10 \mathrm{mg} / \mathrm{m}^{2}$ and melphalan $5 \mathrm{mg} / \mathrm{m}^{2}$ on day 1 , and bortezomib $0.7 \mathrm{mg} / \mathrm{m}^{2}$ on days 1,4 , 8 and 11, on a 28-day cycle. A maximum of 6 cycles are planned. At the time of publication, 1 patient had achieved nCR and 2 patients had stable disease. No DLTs were reported, though 1 patient had transient grade 3 neutropenia. ${ }^{53}$ Further updates of this study are necessary before conclusions may be drawn about this regimen.

\section{Conclusions}

The management of MM has changed rapidly over the past 10 years with increased understanding of the biology of the ubiquitin proteasome pathway, mechanisms of chemoresistance to this pathway, and methods of targeting it for treatment. Combination therapy with PLD+B is an important therapeutic option for patients with relapsed or refractory myeloma that provides superior outcomes to single-agent bortezomib, including improved 15 -month OS rates. As patients with relapsed or refractory MM have often previously received anthracyclines, thalidomide, and/or autologous stem cell transplant, it is significant that PLD+B improves patient outcomes irrespective of these prior exposures. The lack of further benefit seen with adding PLD to bortezomib in patients with low $\beta_{2}$-microglobulin and deletion of chromosome 13 suggest that bortezomib may be solely responsible for the responses seen in these subgroups of patients.

The tolerability profile of PLD+B is noteworthy. Treatment emergent peripheral neuropathy is a common clinical challenge in patients with MM. That PLD is able to improve patient outcomes without exacerbating symptoms of neuropathy adds to its usefulness. This steroid-sparing combination is also a valuable choice for patients with poorly controlled diabetes mellitus and those who are otherwise steroid intolerant. It is equally important that in a disease with a median age at diagnosis of 70 years, this combination does not increase the risk of cardiotoxicity compared with bortezomib alone. ${ }^{54}$ Finally, the lack of need for prophylactic anticoagulation, which is in contrast to immunomodulatory derivative $\left(\mathrm{Imid}^{\circledR}\right.$ )-steroid combinations, is advantageous for patients at risk for falls, or with other bleeding tendencies. While these advantages are important, clinicians must be vigilant for the gastrointestinal toxicities, neutropenia, stomatitis, and hand-foot-syndrome that can be seen with $\mathrm{PLD}+\mathrm{B}$, so that these may be promptly addressed if they develop.

Ongoing phase II studies are currently evaluating the combination of PLD+B with such agents as dexamethasone, thalidomide, and melphalan in the relapsed/refractory setting. These trials have a limited number of patients and follow-up to date; however mature data may suggest additional treatment options in the future.

Because of its significant activity in the relapsed/ refractory setting, with manageable and non-overlapping toxicities, PLD+B is also being evaluated as induction therapy prior to ASCT. This Cancer and Leukemia Group B (CALBG) study led by Orlowski et al enrolled 63 patients with symptomatic MM requiring therapy on the same B-PLD schema utilized in the phase 3 study. Preliminary response data on 29/63 patients demonstrated a $\mathrm{CR} / \mathrm{nCr}$ rate of $28 \%$ and ORR of $79 \%$. Stem cell collection, in a limited 
number of evaluable patients, was not compromised. ${ }^{55}$ If longer follow-up confirms these promising results, this steroid-sparing combination may provide an effective option for patients with improved tolerability over other currently used regimens.

In future clinical trials of PLD+B, it will be useful to assess whether fluorescence in situ hybridization (FISH), conventional cytogenetics, and/or molecular profiling can be used to determine which patients are most likely to benefit from this regimen.

\section{Acknowledgments}

Support: RZO, a Leukemia and Lymphoma Society Mansbach Foundation Scholar in Clinical Research, would like to acknowledge support from the Leukemia and Lymphoma Society (6096-07), the Multiple Myeloma Research Foundation, and the National Cancer Institute (RO1 CA102278).

\section{Disclosures}

The authors disclose no conflicts of interest.

\section{References}

1. Kane RC, Bross PF, Farrell AT, et al. Velcade: US FDA approval for the treatment of multiple myeloma progressing on prior therapy. Oncologist. 2003;8(6):508-513.

2. Kane RC, Farrell AT, Sridhara R, et al. United States Food and Drug Administration approval summary: bortezomib for the treatment of progressive multiple myeloma after one prior therapy. Clin Cancer Res. 2006;12(10):2955-60.

3. Richardson PG, Barlogie B, Berenson J, et al. A phase 2 study of bortezomib in relapsed, refractory myeloma. [see comment]. New Engl J Med. 2003;348(26):2609-2617.

4. Richardson PG, Sonneveld P, Schuster MW, et al. Bortezomib or high-dose dexamethasone for relapsed multiple myeloma. [see comment]. New Engl J Med. 2005;352(24):2487-2498.

5. Richardson PG, Sonneveld P, Schuster MW, et al. Extended follow-up of a phase 3 trial in relapsed multiple myeloma: final time-to-event results of the APEX trial. Blood. 2007;110(10):3557-3560.

6. Alberts DS, Salmon SE. Adriamycin (NSC-123127) in the treatment of alkylator-resistant multiple myeloma: a pilot study. Cancer Chemother Rep. 1975;59(2 Pt 1):345-350.

7. Alexanian R, Barlogie B, Tucker S. VAD-based regimens as primary treatment for multiple myeloma. Am J Hematol. 1990;33(2):86-89.

8. Anderson H, Scarffe JH, Ranson M, et al. VAD chemotherapy as remission induction for multiple myeloma. $\mathrm{Br} J$ Cancer. 1995;71(2):326-330.

9. Barlogie B, Smith L, Alexanian R. Effective treatment of advanced multiple myeloma refractory to alkylating agents. $N$ Engl J Med. 1984;310(21):1353-1356.

10. Cavo M, Zamagni E, Tosi P, et al. Superiority of thalidomide and dexamethasone over vincristine-doxorubicin-dexamethasone (VAD) as primary therapy in preparation for autologous transplantation for multiple myeloma. Blood. 2005;106(1):35-39.

11. Segeren CM, Sonneveld P, van der Holt B, et al. Vincristine, doxorubicin and dexamethasone (VAD) administered as rapid intravenous infusion for first-line treatment in untreated multiple myeloma. Br J Haematol. 1999;105(1):127-130.
12. Rifkin RM, Gregory SA, Mohrbacher A, et al. Pegylated liposomal doxorubicin, vincristine, and dexamethasone provide significant reduction in toxicity compared with doxorubicin, vincristine, and dexamethasone in patients with newly diagnosed multiple myeloma: a Phase III multicenter randomized trial. Cancer. 2006;106(4):848-858.

13. Zervas K, Dimopoulos MA, Hatzicharissi E, et al. Primary treatment of multiple myeloma with thalidomide, vincristine, liposomal doxorubicin and dexamethasone (T-VAD doxil): a phase II multicenter study. Ann Oncol. 2004;15(1):134-138.

14. Ferrazzi E, Woynarowski JM, Arakali A. et al. DNA damage and cytotoxicity induced by metabolites of anthracycline antibiotics, doxorubicin and idarubicin. Cancer Commun. 1991;3(6):173-180.

15. Foglesong PD, Reckord C, Swink S. Doxorubicin inhibits human DNA topoisomerase I. Cancer Chemother Pharmacol. 1992;30(2):123-125.

16. Facchinetti T, Mantovani A, Cantoni L, Cantoni R, Salmona M. Intercalation with DNA is a prerequisite for daunomycin, adriamycin and its congeners in inhibiting DNAase I. Chem Biol Interact. 1978;20(1):97-102.

17. Fornari FA, Randolph JK, Yalowich JC, Ritke MK, Gewirtz DA. Interference by doxorubicin with DNA unwinding in MCF-7 breast tumor cells. Mol Pharmacol. 1994;45(4):649-656.

18. Momparler RL, Karon M, Siegel SE, Avila F. Effect of adriamycin on DNA, RNA, and protein synthesis in cell-free systems and intact cells. Cancer Res. 1976;36(8):2891-2895.

19. Eliot H, Gianni L, Myers C. Oxidative destruction of DNA by the adriamycin-iron complex. Biochemistry. 1984;23(5):928-936.

20. Myers C, Gianni L, Zweier J, Muindi J, Sinha BK, Eliot H, et al. Role of iron in adriamycin biochemistry. Fed Proc. 1986;45(12): 2792-2797.

21. Adams J, Palombella VJ, Sausville EA, et al. Proteasome inhibitors: a novel class of potent and effective antitumor agents. Cancer Res. 1999;59(11):2615-2622.

22. Orlowski RZ. The role of the ubiquitin-proteasome pathway in apoptosis. Cell Death Differ. 1999;6(4):303-313.

23. Hideshima $\mathrm{T}$, Chauhan D, Richardson $\mathrm{P}$, et al. NF-kappaB as a therapeutic target in multiple myeloma. J Biol Chem. 2002;277(19): 16639-16647.

24. Hideshima T, Mitsiades C, Akiyama M, et al. Molecular mechanisms mediating antimyeloma activity of proteasome inhibitor PS-341. Blood. 2003;101(4):1530-1534.

25. Mitsiades N, Mitsiades CS, Poulaki V, et al. Molecular sequelae of proteasome inhibition in human multiple myeloma cells. Proc Natl Acad Sci U S A. 2002;99(22):14374-14379.

26. Rajkumar SV, Richardson PG, Hideshima T, et al. Proteasome inhibition as a novel therapeutic target in human cancer. J Clin Oncol. 2005;23(3):630-639.

27. Ambudkar SV, Kimchi-Sarfaty C, Sauna Z, et al. P-glycoprotein: from genomics to mechanism. Oncogene. 2003;22(47):7468-7485.

28. Hideshima T, Richardson $\mathrm{P}$, Chauhan $\mathrm{D}$, et al. The proteasome inhibitor PS-341 inhibits growth, induces apoptosis, and overcomes drug resistance in human multiple myeloma cells. Cancer Res. 2001;61(7):3071-3076.

29. Ma MH, Yang HH, Parker K, et al. The proteasome inhibitor PS-341 markedly enhances sensitivity of multiple myeloma tumor cells to chemotherapeutic agents. Clin Cancer Res. 2003;9(3):1136-1144.

30. Mitsiades N, Mitsiades CS, Richardson PG, et al. The proteasome inhibitor PS-341 potentiates sensitivity of multiple myeloma cells to conventional chemotherapeutic agents: therapeutic applications. Blood. 2003;101(6):2377-2380.

31. Orlowski RZ, and Baldwin Jr AS. NF-kappaB as a therapeutic target in cancer. Trends Mol Med. 2002;8(8):385-389.

32. Wang CY, Mayo MW, Baldwin AS Jr TNF- and cancer therapy-induced apoptosis: potentiation by inhibition of NF-kappaB. [see comment]. Science. 1996;274(5288):784-787. 
33. Fujita T, Washio K, Takabatake D, et al. Proteasome inhibitors can alter the signaling pathways and attenuate the P-glycoprotein-mediated multidrug resistance. Int J Cancer. 2005;117(4):670-682.

34. Small GW, Shi YY, Edmund NA, et al. Evidence that mitogen-activated protein kinase phosphatase-1 induction by proteasome inhibitors plays an antiapoptotic role. Mol Pharmacol. 2004;66(6):1478-1490.

35. Orlowski RZ, Voorhees PM, Garcia RA, et al. Phase I trial of the proteasome inhibitor bortezomib and pegylated liposomal doxorubicin in patients with advanced hematologic malignancies. Blood. 2005;105(8):3058-3065.

36. Biehn SE, Moore DT, Voorhees PM, et al. Extended follow-up of outcome measures in multiple myeloma patients treated on a phase I study with bortezomib and pegylated liposomal doxorubicin. Ann Hematol. 2007;86(3):211-216.

37. Orlowski RZ, Nagler A, Sonneveld P, et al. Randomized phase III study of pegylated liposomal doxorubicin plus bortezomib compared with bortezomib alone in relapsed or refractory multiple myeloma: combination therapy improves time to progression. J Clin Oncol. 2007;25(25):3892-3901.

38. Ning YM, Nagher R, Sridhara R, et al. Liposomal doxorubicin in combination with bortezomib for relapsed or refractory multiple myeloma. Oncology (Williston Park). 2007;21(12): 1503-1508; discussion 1511, 1513, 1516 passim.

39. Blade J, Samson D, Reece D, et al. Criteria for evaluating disease response and progression in patients with multiple myeloma treated by high-dose therapy and haemopoietic stem cell transplantation. $\mathrm{Br} J$ Haematol. 1998;102(5):1115-1123.

40. Braccalenti G, Ferrara P, Gubbiotti M, et al. Antitumor activity of bortezomib-pegylated liposomal doxorubicine association as salvage therapy in multiple myeloma patients. Blood. 2007;110:4832.

41. San Miguel J, Hajek R, Nagler A, et al. Doxil + velcade in previously treated myeloma patients aged $>65$. Haematologica. 2007;97(6 Suppl 2):159 abstract \#620.

42. Hajek R, Nagler A, Sonneveld P, et al. Doxil + velcade in previously treated high risk myeloma. Haematologica. 2007;97(6 Suppl 2):162 abstract \#629.

43. Nagler A, Sonneveld P, Spencer A, et al. Doxil + velcade in previously treated myeloma patients with prior stem cell transplant. Haematologica. 2007;97(6 Supp1 2):161 abstract \#625.

44. Blade J, San Miguel J, Nagler A, et al. The prolonged time to progression with pegylated liposomal doxorubicin + bortezomib versus bortezomib alone in relapsed or refractory multiple myeloma is unaffected by extent of prior therapy or previous anthracycline exposure. Blood. 2007;110(11):410.
45. Sonneveld P, Hajek R, Nagler A, et al. Combined pegylated liposomal doxorubicin and bortezomib is highly effective in patients with recurrent or refractory multiple myeloma who received prior thalidomide/ lenalidomide therapy. Cancer. 2008;112(7):1529-15237.

46. Sutherland HJ, Blade J, San Miguel J, et al. Effect of disease stage and time since diagnosis on time to progression for pegylated liposomal doxorubicin + bortezomib vs bortezomib alone in relapsed or refractory multiple myeloma. Blood. 2007;110(11):2740.

47. Kumar S, Blade J, San Miguel J, et al. Pegylated liposomal doxorubicin (PLD) in combination with bortezomib (B) may provide therapeutic advantage for high-risk multiple myeloma patients relapsing within 12 months of stem cell transplant. Blood. 2007;110(11):2730.

48. Jakubowiak AJ, Friedman J, Kendall T, et al. A phase II study of combination of bortezomib, liposomal doxorubicin, and dexamethasone (VDD) as first line therapy for multiple myeloma. $J$ Clin Oncol. 2006 ASCO Annual Meeting Proceedings Part I, 2006;24(18S):17504.

49. Offidani M, Bringhen S, Corvatta L, et al. Thalidomide-dexamethasone plus pegylated liposomal doxorubicin vs thalidomide-dexamethasone: a case-matched study in advanced multiple myeloma. Eur J Haematol. 2007;78(4):297-302.

50. Duryea BGM, Harousseau JL, Miguel JS, et al. International uniform response criteria for multiple myeloma. Leukemia. 2006;20(9):1467-1473.

51. Offidani M, Polloni C, Corvatta L, et al. ThaDD-V treatment for patients with relapsed/refractory multiple myeloma: a feasibility/activity study. Blood. 2007;110(11):2729.

52. Chanan-Khan AA, Padmanabhan S, Miller KC, et al. Final results of a phase II study of bortezomib (Velcade) in combination with liposomal doxorubicin (Doxil) and thalidomide (VDT) demonstrate a sustained high response rates in patients (pts) with relapsed (rel) or refactory (ref) multiple myeloma. Blood. 2006;108:3539.

53. Chari A, Kaplan L, Linker C, et al. Phase I/II study of bortezomib in combination with liposomal doxorubicin and melphalan in relapsed or refractory multiple myeloma. Blood. 2005;106:5182.

54. Ries LAG, Melbert D, Krapcho M, et al. (eds). SEER Cancer Statistics Review, 1975-2005, National Cancer Institute. Bethesda, MD, SEER Cancer Statistics Review, 1975-2005, National Cancer Institute. Bethesda, MD, http://seer.cancer.gov/csr/1975_ 2005/, 2007.

55. Orlowski RZ, Peterson BL, Sanford B, et al. Bortezomib and pegylated liposomal doxorubicin as induction therapy for adult patients with symptomatic multiple myeloma: Cancer and Leukemia Group B Study 10301. Blood. 2006;108(11):797. 
